Classification

Physics Abstracts

$05.40-42.10-82.70$

\title{
Brownian dynamics in a confined geometry. Experiments and numerical simulations
}

\author{
N. Garnier and N. Ostrowsky \\ Laboratoire de Physique de la Matière Condensée ( ${ }^{*}$ ) \\ Université de Nice Sophia Antipolis, 06034 Nice Cedex, France
}

(Received 18 April 1991, accepted 17 July 199I)

\begin{abstract}
Résumé. - La dynamique brownienne de particules colloïdales au voisinage immédiat d'une paroi rigide est mesurée par la technique de diffusion quasi-élastique de la lumière en onde évanescente. On observe un net décroissement du coefficient de diffusion, dû au ralentissement hydrodynamique des particules très proches de la paroi. Cet effet est d'autant plus marqué que les particules peuvent se rapprocher très près de la paroi, c'est-à-dire que la portée de la répulsion statique paroi/particule est faible. Il est donc possible de tester les interactions statiques paroi/particules via une expérience de diffusion dynamique de la lumière. Les données sont analysées par une simulation de dynamique Brownienne, particulièrement adaptée à l'interprétation des résultats de diffusion de la lumière par des diffuseurs " gênés », tels que des particules confinées au voisinage d'une paroi, ou piégées dans des milieux poreux ou des gels.
\end{abstract}

\begin{abstract}
The Brownian dynamics of a colloidal suspension is measured in the immediate vicinity of a rigid surface by the Evanescent Quasielastic Light Scattering Technique. A net decrease of the measured diffusion coefficient is observed, due to the hydrodynamic slowing down of the particles very close to the wall. This effect is all the more important when the particles are allowed to get closer to the wall, i.e. when the range of the static wall/particle repulsive interaction decreases. It thus provides a mean for testing the particle/wall static interactions via a dynamic light scattering measurement. The data are analysed by a Brownian dynamic simulation which is proven to be quite valuable to interpret light scattering data from " hindered " scatterers, such as particles confined in the neighbourhood of a wall or trapped in a porous media or a gel.
\end{abstract}

\section{Introduction.}

The interactions between particles suspended in a liquid and the solid wall confining the suspension are at the basis of a number of important practical phenomena such as particle sedimentation and adhesion on a substrate.

These interactions may be classified in two types:

- The static interactions mainly include the Van der Waals attraction and the electrostatic interactions between the charges carried by the particles and the solid wall. The steric

(*) CNRS URA 190. 
interactions of entropic origin, present when the interacting surfaces are flexible (for example, undulating vesicles or particles coated with a polymer layer) may be neglected in our problem. This type of interactions lead to a non-uniform concentration profile in the vicinity of the wall.

- The hydrodynamic interactions due to the fact that the liquid flow created by a moving particle is perturbed by the presence of the wall and thus reacts back onto the motion of the original particle.

A number of theoretical [1] and numerical [2] studies have explicited the form and numerical values of these interactions and more recently some molecular dynamics simulations $[3,4]$ have helped understand their role in the Brownian motion of particles in the immediate vicinity of a rigid wall.

As far as experiments go, the problem is far less advanced. Macroscopic experiments have monitored the fall of suspended balls onto a solid surface, thus measuring the friction coefficient $\Lambda(z)$ as a function of the distance $z$ between the particle and the wall [5]. On a more microscopic scale (particles around $10 \mu \mathrm{m}$ in diameter) static experiments have studied the height distribution of suspended particles above a given transparent plate, from which the static interaction potential between the particles and the wall can be deduced [6]. Static Fluorescence Techniques have also been used to measure the concentration profile of particles doped with fluorescent probes in the vicinity of a transparent wall $[7,8]$.

The purpose of this paper is to give some new experimental results on the Brownian dynamics of particles close to a surface, measured by an original method developed in our laboratory: The quasi elastic light scattering technique using as the incident light an evanescent wave which thus only probes particles within a distance less than the penetration depth of the wave (see Sect. 2). To analyse our data, we have performed a " computer simulated light scattering experiment " (see Sect. 3) to generate the correlation function of the electric field scattered by a Brownian particle submitted to both the static and hydrodynamic interactions discussed above.

\section{Evanescent Q.L.S. experiment.}

\subsection{EXPERIMENTAL CONDITIONS.}

Material : We have studied an aqueous latex suspension (particle diameter $=0.09 \mu \mathrm{m}$ ) whose concentration $\left(c \cong 3 \times 10^{-4} \mathrm{~g} / \mathrm{cm}^{3}\right.$, i.e. mean distance between particle $\left.=1 \mu \mathrm{m}\right)$ is large enough to provide a confortable signal in the evanescent wave regime, but low enough to allow us to neglect the particle/particle interactions. We have used different salt concentrations so as to be able to partially screen the electrostatic repulsion between the suspended particles and the glass wall, both negatively charged.

Optical set-up (see Fig. 1) : The liquid sample is contained in a half cylindrical cell, sealed by an optically flat surface (polished to $\lambda / 20$ ) of a larger semi-cylindrical glass prism. This surface was ultrasonically cleaned with distilled water after each experiment. The sample holder is placed on a precision turntable, so as to easily change the incident angle $\theta_{i}$ of the vertically polarized Argon laser $(300 \mathrm{~mW}$ at $\lambda=514.5 \mathrm{~nm})$. The critical angle for total reflection is given by the usual relation : $\sin \theta_{\mathrm{c}}=n_{\text {Liquid }} / n_{\mathrm{Glass}}$. For $\theta_{\mathrm{i}}>\theta_{\mathrm{c}}$, the incident wave vector in the medium has a real component $k_{\mathrm{i}}=2 \pi n_{\mathrm{Glass}} / \lambda$ parallel to the flat surface of the prism, and an imaginary component equal to the inverse of the penetration depth $\xi$ given by:

$$
\xi=\left(\lambda / 2 \pi n_{\mathrm{Glass}}\right)\left[\sin ^{2} \theta_{\mathrm{i}}-\sin ^{2} \theta_{\mathrm{c}}\right]^{-1 / 2}
$$

This means that we are conducting a light scattering experiment with an incident beam always 


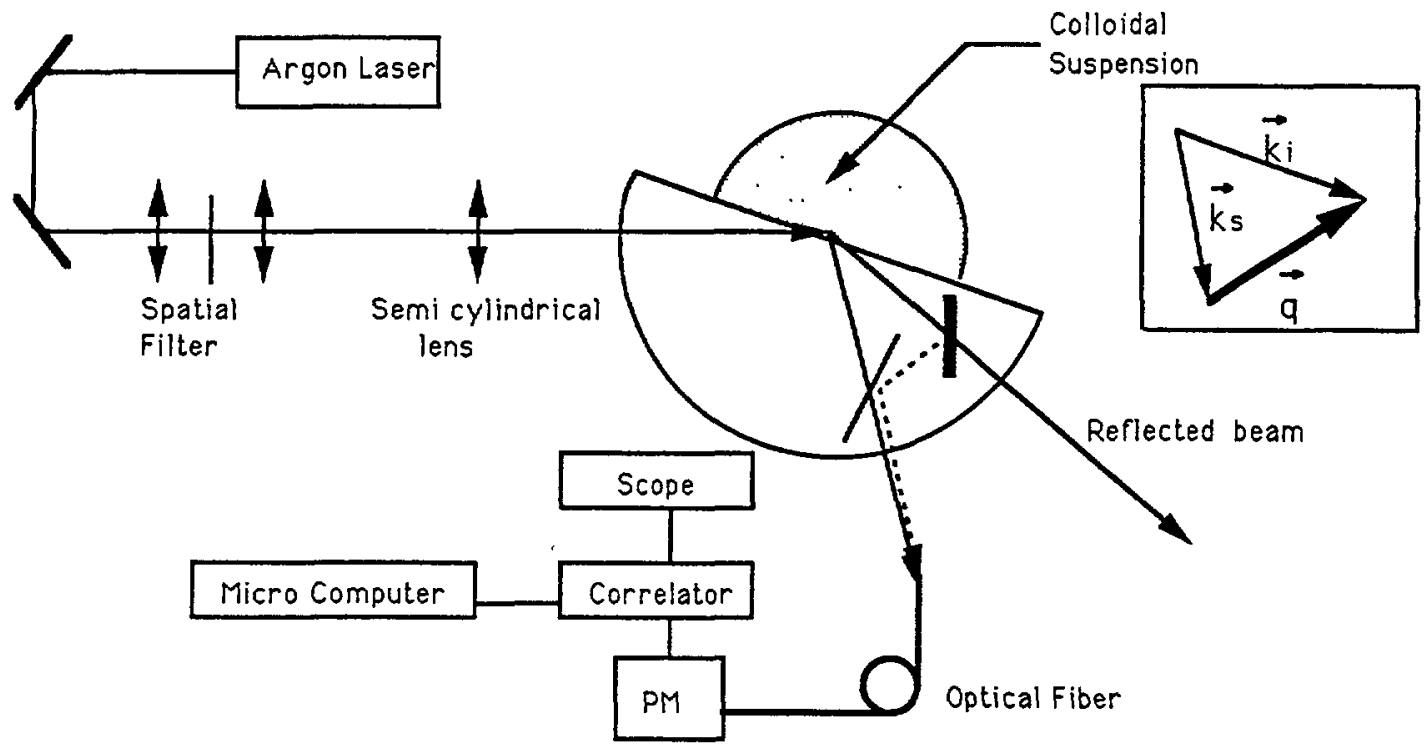

Fig. 1. - The experimental set-up.

parallel to the flat glass/liquid interface and whose intensity decreases exponentially as $\mathrm{e}^{-2 z / \xi}$ with the distance $z$ from the wall.

The light scattered in the liquid suspension at an angle $\theta$ from the incident wave vector $\mathbf{k}_{i}$ is collected via an optical fiber onto a photomultiplier whose output is analyzed with the standard correlation techniques. To ensure $100 \%$ heterodyning, an adjustable fraction of the reflected laser beam was made to coincide with the path of the scattered light and thus mixed on the photomultiplier surface.

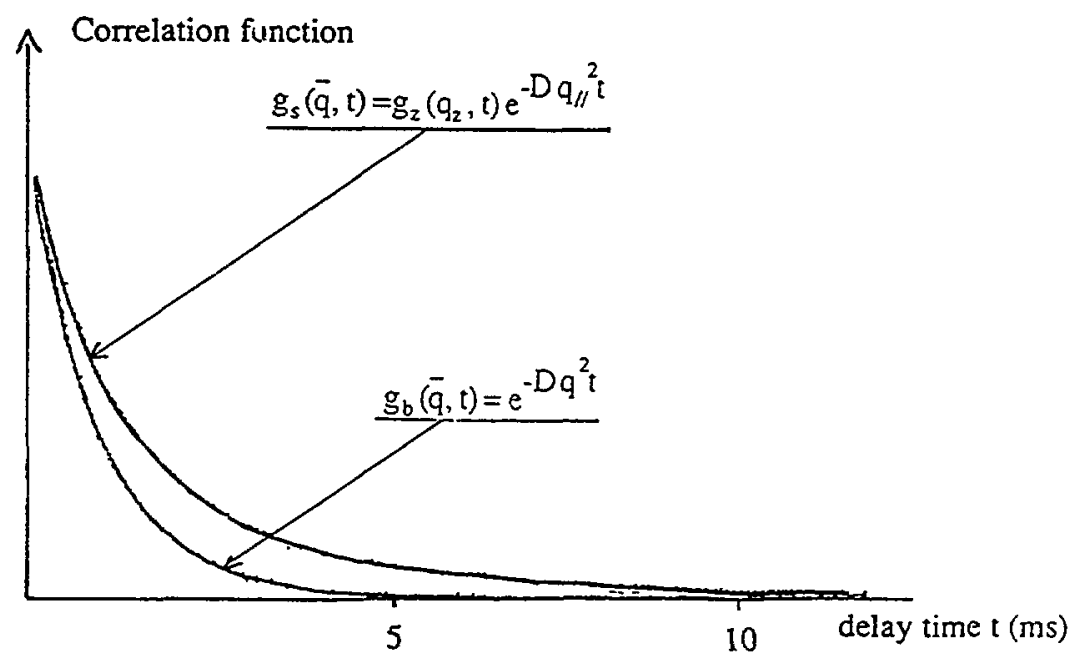

Fig. 2. - Typical bulk $\left[g_{\mathrm{b}}(q, t)\right]$ and surface $\left[g_{\mathrm{s}}(q, t)\right]$ correlation functions, measured for the same scattering angle $\theta$, i.e. the same scattering vector $q$. The different time behaviours are well accounted for by expressions (5) and (8). 
2.2 EXPERIMENTALLY MEASURED CORRELATION FUNCTIONS. - Typical correlation functions recorded for $\theta_{\mathrm{i}}$ just below $(\xi=0.8 \mu \mathrm{m})$ and just above $(\xi=\infty)$ the critical angle $\theta_{c}$, but at the same scattering angle $\theta$, are shown in figure 2, illustrating the so-called «surface » and «bulk» correlation functions whose theoretical expressions are now recalled.

Bulk correlation function: In a heterodyne experiment, the intensity correlation function is a linear function of the first order correlation function of the scattered electric field, which, with homogenous illumination of the scattering volume and in its normalized from, can be written as :

$$
g(t)=\operatorname{Re}\left\langle e^{i q \cdot r(t)}\right\rangle \text { where the scattering wave vector } \mathbf{q}=\mathbf{k}_{\mathrm{i}}-\mathbf{k}_{\mathrm{s}}
$$

is the difference between the incident $\left(\mathbf{k}_{\mathrm{j}}\right)$ and the scattered $\left(\mathbf{k}_{\mathrm{s}}\right)$ wave vectors, and $\mathbf{r}(t)$ is the vector position of the particle which was at the origin at time zero. The average is to be taken on a great number of independent Brownian particles.

In the case of the usual bulk geometry, the probability density for a particle to be at time $t$ at a distance $r$ from its starting point at time zero is the well known Gaussian probability :

$$
P(r, t)=(4 \pi D t)^{-3 / 2} \exp \left(-r^{2} / 4 D t\right)
$$

where $D=k T / 6 \pi \eta R$ is the bulk diffusion coefficient, related to the particle radius $R$ and the suspension's viscosity $\eta$. Using this probability density to compute the average in equation (2) leads to the usual expression for the bulk correlation function:

$$
\begin{aligned}
g_{\mathrm{b}}(t) & =\int \mathrm{e}^{i_{\mathrm{q}} \cdot \mathrm{r}(t)} P(r, t) \mathrm{d}^{3} r \\
& =\exp \left(-D q^{2} t\right) .
\end{aligned}
$$

Surface correlation function: In the presence of a wall, it is useful to decompose the 3D Brownian motion into two independent motions, one parallel and one perpendicular to the wall, along the direction $\mathrm{Oz}$. Neglecting, to begin with, the particles/wall interactions, the first motion obeys the usual 2D Brownian motion statistics, and the second one includes the "mirror" effect of the wall. Accordingly, the proper probability density to be used in computing the average in equation (2) is no longer given by equation (3) but now reads :

$$
P\left(r_{\|}, z, z_{0}, t\right)=(4 \pi D t)^{-3 / 2} \mathrm{e}^{-\frac{r^{2}}{4 D t}}\left[\mathrm{e}^{-\frac{\left(z-z_{0}\right)^{2}}{4 D t}}+\mathrm{e}^{-\frac{\left(z+z_{0}\right)^{2}}{4 D t}}\right]
$$

where $\left(0, z_{0}\right)$ and $(\eta, z)$ are the parallel and perpendicular coordinates of the particle at time 0 and $t$, respectively (see Fig. 5).

In the evanescent wave geometry, one must further take into account the fact that the scattering volume is not uniformly illuminated, which requires that the average in equation (2) be properly weighted by the electric field amplitude at the particle's position at time 0 $\left[E_{0} \mathrm{e}^{-z_{0} / \xi}\right]$ and time $t\left[E_{0} \mathrm{e}^{-z / \xi}\right]$.

The theoretical form of the "surface» correlation function $g_{s}(t)$ is thus given by the following integral :

$$
g_{\mathrm{s}}(t)=\int \frac{\mathrm{d} z_{0}}{\xi} \mathrm{e}^{-\frac{z_{0}}{\xi}} \iiint P\left(\boldsymbol{r}_{\|}, z, z_{0}, t\right) \mathrm{e}^{-\frac{z}{\xi}} \mathrm{e}^{i q_{\|} \eta} \mathrm{e}^{i q_{z}\left(z-z_{0}\right)} \mathrm{d}^{2} \eta_{\|} \mathrm{d} z
$$


which has been found [9] to be equal to :

$$
g_{\mathrm{s}}(t)=\exp \left(-D q_{\|}^{2} t\right) g_{z}\left(q_{z}, \xi, D, t\right)
$$

where $q_{\|}$and $q_{z}$ are the components of the scattering vector parallel and perpendicular to the wall, and $g_{z}\left(q_{z}, \xi, D, t\right)$ is an analytical function whose limited expansion can easily be implementd on a micro-computer.

The theoretical correlation functions (5) and (8) have been used to draw the solid lines in figure 2 , leaving as an adjustable parameter the diffusion coefficient $D$. Now, the value found for the best adjustment of the surface correlation function was always lower than the value found for the bulk measurement, the difference being all the greater when the particles were allowed to come closer to the wall, i.e. when the particles/wall repulsion was reduced by increasing the salt concentration of the suspension. This is interpreted as the combined effect of the repulsive hydrodynamic and electrostatic interactions between the particles and the wall as we now discuss.

2.3 Role OF PARTICles/WALl INTERACTIONS. - As mentioned in the introduction, two kinds of interactions must be considered.

The static particle/wall interactions represented by a position dependent potential interaction $U(z)$, results in a non-uniform distribution of particles in the vicinity of the wall. As we studied negatively charged latex particles of radius $R$ suspended in salty water, in the vicinity of a glass wall, both negatively charged, the electrostatic interaction may be derived from the DLVO theory $[10]$ :

$$
\begin{aligned}
U_{\mathrm{el}}(s)=\frac{\varepsilon R(R+s)}{2(2 R+s)}\left[\left(\psi_{1}^{2}+\psi_{2}^{2}+\right.\right. & \left.H \psi_{1} \psi_{2}\right) \ln \left[1+\left(\frac{R}{R+s}\right)^{1 / 2} \mathrm{e}^{-\kappa s}\right]+ \\
& \left.+\left(\psi_{1}^{2}+\psi_{2}^{2}-H \psi_{1} \psi_{2}\right) \ln \left[1-\left(\frac{R}{R+s}\right)^{1 / 2} \mathrm{e}^{-\kappa s}\right]\right]
\end{aligned}
$$

with the following notation:

$s$ is closest distance between the particle's surface and the wall (i.e. : $s=z-R$ )

$\kappa^{-1}$ is the screening Debye length :

$$
\kappa^{-1}=\left[\varepsilon k T /\left(2 e^{2} N_{\mathrm{A}} c_{\mathrm{salt}}\right)\right]^{1 / 2}
$$

(with $N_{\mathrm{A}}$. the Avogadro number, $\varepsilon:$ the dielectric constant of the medium, $e$ : the electronic charge and $c_{\text {salt }}$. the salt concentration).

$\psi_{1}$ and $\psi_{2}$ are the surface potentials of the latex particle and the glass wall (typically on the order of $50 \mathrm{mV}$ ).

$H$ is a dimensionless quantity defined as :

$$
H=\left(\frac{R}{R+s}\right)^{1 / 2}+\left(\frac{R+s}{R}\right)^{1 / 2}
$$

To this repulsive potential must be added the attractive Van der Waals potential derived from Hamaker's theory [11]:

$$
U_{\mathrm{vw}}(s)=-\frac{A}{6}\left[\frac{R}{s}+\frac{R}{s+2 R}+\ln \left(\frac{s}{s+2 R}\right)\right]
$$

with $A \cong 1 k T$.

The resulting interaction potential $U(s)$ is shown in figure $3 \mathrm{a}$ for different salt concentrations, and the corresponding Boltzman concentration profiles $c(s)$ are plotted in figure $3 \mathrm{~b}$. 

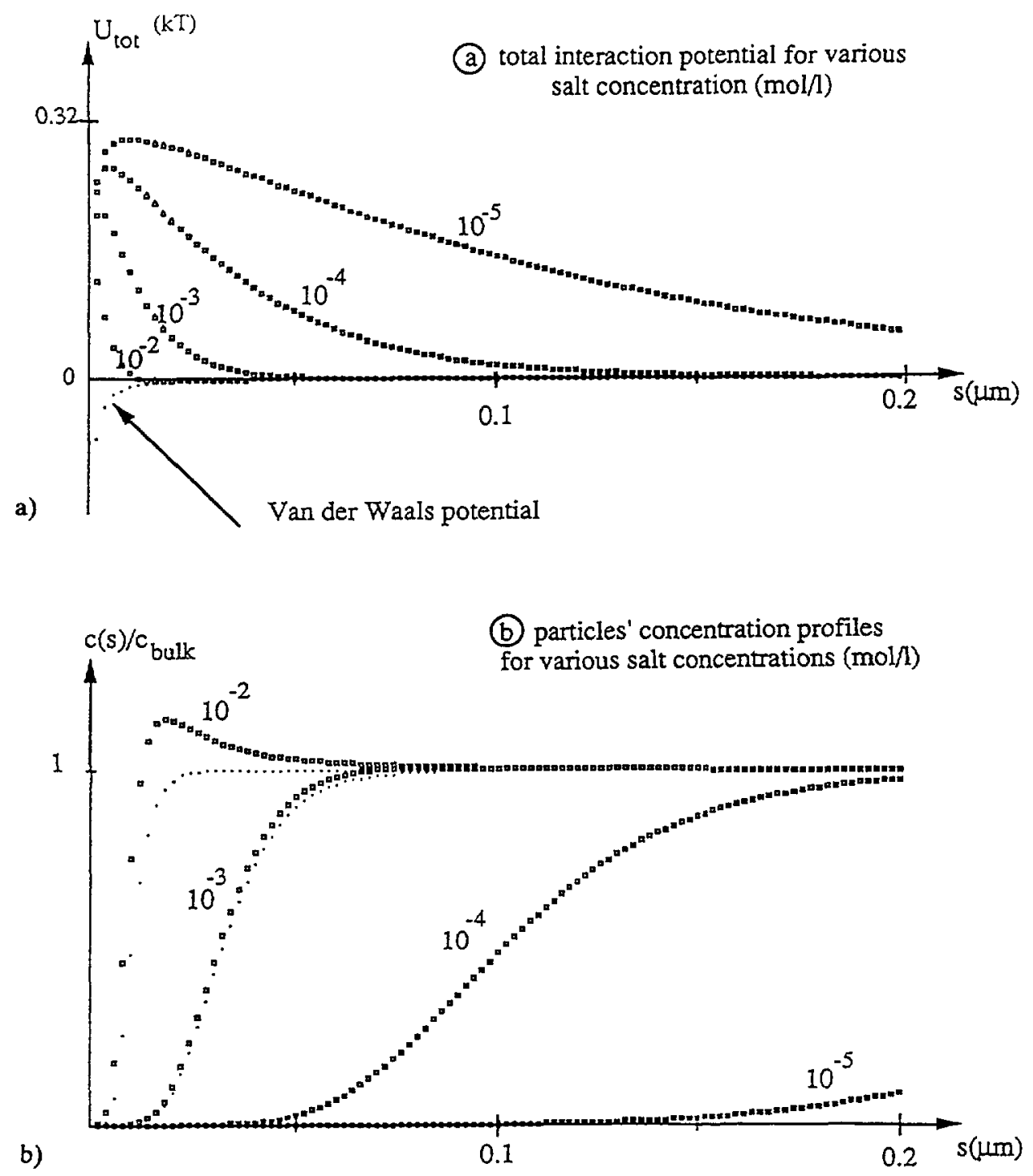

Fig. 3. - Part a) Total Interaction potential (squares) between a particle and the glass wall, both negatively charged, for various salt concentrations. The attractive Van der Waals part of the potential is shown with the dotted line. Part b) Corresponding particles' Boltzman concentration profiles (squares). The profiles obtained when ignoring the Van der Waals attraction are shown with the dotted lines.

This figure clearly shows that the effect of the Van der Waals attractive potential will only be detectable for the highest salt concentration used $\left(10^{-2} \mathrm{~mol} / \mathrm{l}\right)$ and the shortest penetration depth $(\xi=0.2 \mu \mathrm{m})$, enhancing by $10 \%$ the hydrodynamic slowing down as will be explained in the next section. This attractive potential was however important in all our experiments, as it was responsible for a very slow but detectable aggregation, all the more rapid when the salt concentration was increased. Beyond $10^{-2} \mathrm{~mol} / \mathrm{l}$, the aggregation was found to be too rapid to insure good evanescent wave conditions on the flat surface of the prism during the data acquisition time (of the order of $10 \mathrm{~min}$ ). 
The effect of the hydrodynamic interactions between the particles and the wall, mediated by the water molecules, are included through a position-dependent friction tensor $\Lambda$ which, multiplied by the vector velocity $V$ of the particle, yields the friction force $F$ experimented by the particle :

$$
\left(\begin{array}{l}
F_{\|} \\
F_{z}
\end{array}\right)=\left(\begin{array}{cc}
\Lambda_{\mathbb{}} & 0 \\
0 & \Lambda_{z}
\end{array}\right)\left(\begin{array}{l}
v_{\mathbb{\|}} \\
v_{z}
\end{array}\right)
$$

Using the Einstein Smoluchowsky relation, leads to a position dependent diffusion tensor whose component $D_{\|}(s)$ and $D_{z}(s)$ have been calculated in the literature.

Using Faxen result $[12,4]$, we computed $D_{1}(s)$ with the following limited expansion:

$$
\frac{D_{\|}(s)}{D_{\text {bulk }}}=1-\frac{9}{16}\left(\frac{R}{s+R}\right)+\frac{1}{8}\left(\frac{R}{s+R}\right)^{3}-\frac{45}{256}\left(\frac{R}{s+R}\right)^{4}-\frac{1}{16}\left(\frac{R}{s+R}\right)^{s}+\cdot
$$

$D_{z}(s)$ was derived from Brenner's expression [13,4], keeping the first seven terms of the series :

$$
\begin{aligned}
& \frac{D_{\text {bulk }}}{D_{z}(s)}= \\
& \quad=\frac{4}{3} \sinh \alpha \sum_{n=1}^{\infty} \frac{n(n+1)}{(2 n-1)(2 n+3)}\left[\frac{2 \sinh (2 n+1) \alpha+(2 n+1) \sinh 2 \alpha}{4 \sinh ^{2}(n+1 / 2) \alpha-(2 n+1)^{2} \sinh ^{2} \alpha}-1\right]
\end{aligned}
$$

with $\alpha=\cosh ^{-1}\left(\frac{R+s}{R}\right)$.

To account for this position dependence of the diffusion coefficient in the computation of the correlation function $g_{s}(t)$ is not a trivial matter, except for short times compared to the correlation function relaxation time.

In this limit, a limited expansion of equation (8) yields :

$$
g_{s}^{(1)}(t) \cong\left[1-D_{\|} q_{\|}^{2} t-D_{z}\left(q_{z}^{2}+1 / \xi^{2}\right) t\right] .
$$

For such short times, one can assume that a given scattering Brownian particle is confined to a volume small enough so that its diffusion coefficients $D_{\|}(z)$ and $D_{z}(z)$ can be considered as constant. The observed correlation function is an average of equation (16) over all the Brownian particles contained in the scattering volume. Taking into account the facts that the concentration $c(z)$ of particles near the wall is position dependent (see Fig. 3b) and that particles closer to the wall receive and thus scatter a higher intensity according to the exponential law $\exp (-2 z / \xi)$, leads to the approximation :

$$
\begin{aligned}
g_{\mathrm{s}}^{(1)}(t) & \cong \frac{\int_{0}^{\infty}\left[1-D_{1}(z) q \|^{2} t-D_{z}(z)\left(q_{z}^{2}+1 / \xi^{2}\right) t\right] c(z) \exp (-2 z / \xi) \mathrm{d} z}{\int_{0}^{\infty} c(z) \exp (-2 z / \xi) \mathrm{d} z} \\
& \cong\left[1-\vec{D}(\xi)\left(q^{2}+1 / \xi^{2}\right)\right]
\end{aligned}
$$

thus defining the weighted average $\bar{D}(\xi)$ which has been numerically computed for different penetration depth for $[\mathrm{NaCl}]=10^{-2} \mathrm{~mol} / 1$ (see dotted lines in Fig. 4).

It must be remembered, however, that this approach is only valid for short times, and the above result should be compared with the very beginning of the experimentally measured 


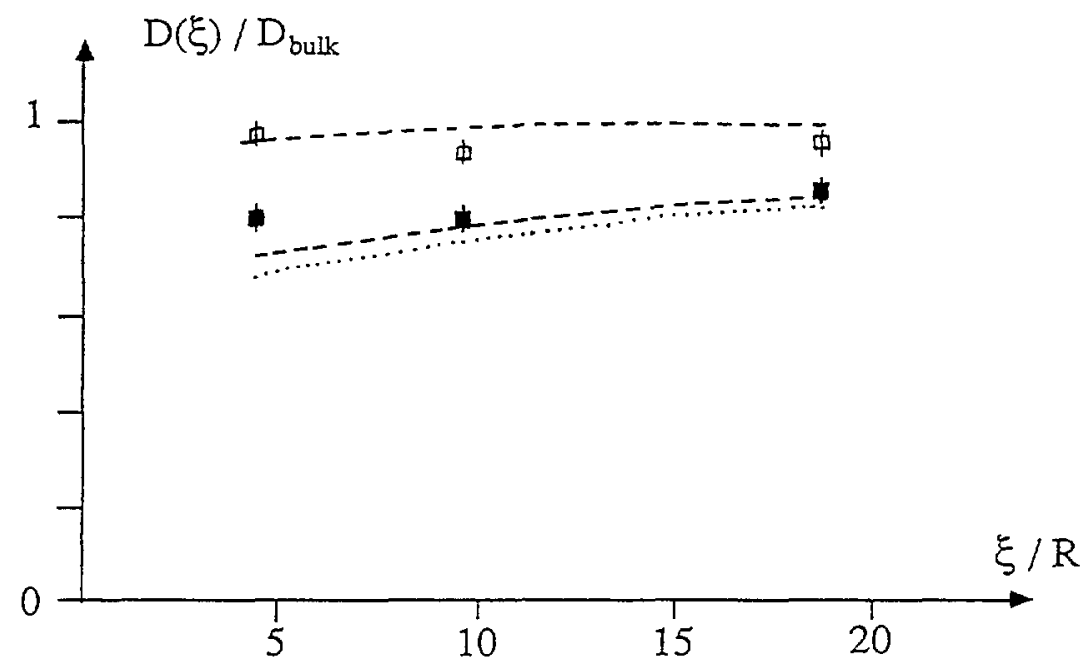

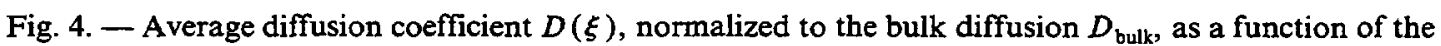
penetration length $\xi$ for two salt concentration $\left(\square: 10^{-2} \mathrm{~mol} / \mathrm{l} ; \boldsymbol{\square}\right.$ : no salt added). The values computed from the "short-time approximation " are shown by the dotted line for $[\mathrm{NaCl}]=10^{-2} \mathrm{~mol} / \mathrm{l}$. The computer simulated results for $[\mathrm{NaCl}]=10^{-2} \mathrm{~mol} / 1$ and $[\mathrm{NaCl}]=10^{-5} \mathrm{~mol} / 1$ are indicated with dashed lines.

correlation function, i.e. its slope at the origin. Unfortunately, this comparison cannot be very precise, as the surface correlation function is far from an exponential and its slope at the origin can only be poorly defined. We thus looked for a better way to analyze our experimental data, which led us to the Brownian dynamics simulations we now describe.

\section{Brownian dynamics simulations.}

The idea is to simulate a light scattering experiment on a computer and derive numerically the expected correlation function $g(t)$. As we explained in section 2.2, any photon correlation experiment measures the normalized correlation function of the electric fields scattered by a walker at time $0, E(0)$, and by the same walker an instant $t$ later, $E(t)$. If the walker at time 0 and $t$ receives the same incident intensity (constant illumination profile), the only difference between $E(0)$ and $E(t)$ is a phase factor $\cos (\mathbf{q} \cdot \mathbf{r}(t))$ where $\mathbf{q}$ is the scattering wave vector and $\mathbf{r}(t)$ is the distance covered by the walker during the time $t$, and thus :

$$
g(t)=\frac{\langle E(t) E(0)\rangle}{\left\langle\left|E(0)^{2}\right|\right\rangle}=\langle\cos (\mathbf{q} \cdot \mathbf{r}(t))\rangle .
$$

If the scattering particle is far from any wall, thus undergoing a Brownian motion with a diffusion constant $D_{b}$, it can be modelled by a random walker which takes every time interval $\tau$ and in any direction a step $\pm\left(2 D_{\mathrm{b}} \tau\right)^{1 / 2}$. The time interval $\tau$ chosen for the simulation must be small compared to the time decay of the Light Scattering correlation function, but large enough to allow for Brownian fluctuations to decay. The light scattering correlation function is then obtained as follows. Let a given walker move around during a time interval $t$, thus covering a distance $\mathbf{r}(t)$; compute the phase factor $\cos (\mathbf{q} \cdot \mathbf{r}(t))$ and repeat the experiment a great number of times, the average of the phase factor progressively building the correlation function $g(t)$. In the case of a free Brownian particle, moving in a constant 

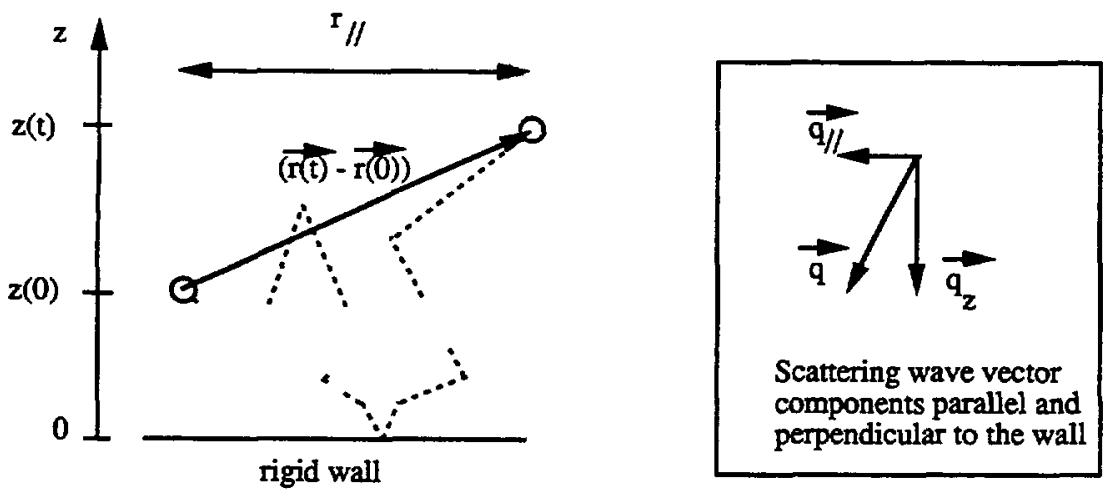

Fig. 5. - Brownian walker in the vicinity of a wall showing the position dependent step and the mirror effect of the wall.

illumination profile, the simulations match the well known analytical result (see Eq. (5)). If we now introduce the exponential illumination profile, together with the mirror effect of the wall (i.e. a walker meeting the wall just bounces back into the suspension see Fig. 5), the simulations exactly match the solution given in equation (8). The method however takes on all its value when we introduce in addition the static and hydrodynamic interactions between the walker and the wall, as we now describe.

Starting from the Langevin equation describing the motion of a Brownian particle submitted to a position-dependent friction coefficient and to a static position-dependent force, Ermak and MacGammon [3] derived the expression for the position-dependent step such a Brownian particle accomplishes. In our problem, the random displacement $L(z)$ of a walker along the $O z$ axis during a time interval $\tau$ is the sum of three terms:

$$
L(z)= \pm\left(2 D_{z}(z) \tau\right)^{1 / 2}+\mathrm{d} D_{z} / \mathrm{d} z \tau+\left[D_{z}(z) / k T\right] F_{z} \tau \text {. }
$$

The first one is the usual random step, $L_{0}= \pm(2 D \tau)^{1 / 2}$ with a $z$ dependent diffusion coefficient $D$. The second one is merely a correction $\mathrm{d} L_{0}$ to the first one, taking into account the fact that $D$ is not constant over the whole step $L_{0}$. This correction term may be simply derived by differentiating $L_{0}$ with respect to $z$ :

$$
\mathrm{d} L_{0}= \pm \frac{1}{2} \frac{2 \frac{\mathrm{d} D}{\mathrm{~d} z} \mathrm{~d} z \tau}{\sqrt{2 D \tau}}
$$

which, for $\mathrm{d} z=L_{0}$, yields the second term of equation (20). The third term of equation (20) is simply the drift of the walker away from the wall, due to the static repulsive force $F_{z}$ computed by taking the gradient of equations (9) and (12).

Now the random displacement $L^{\prime}(z)$ of that same walker parallel to the wall is somewhat simpler as it includes just the random step, whose magnitude however is position dependent :

$$
L^{\prime}(z)= \pm\left(2 D_{\|}(z) \tau\right)^{1 / 2}
$$

We have thus implemented on a MacII microcomputer a program computing the position $\mathbf{r}(t)$ of a walker starting at time zero from a randomly chosen position $\mathbf{r}(0)$, repeating the procedures as the walker moves around. The simulated surface correlation function is 
progressively built up by summing, for each couple of positions $[\mathbf{r}(0), \mathbf{r}(t)]$, the properly weighted phase factor:

$$
\left[g_{\mathrm{s}}(t)\right]_{\text {simulated }}=\frac{1}{N \text { of pairs }} \sum_{\text {over all pars }} \mathrm{e}^{-\frac{z(0)}{\xi}} \mathrm{e}^{-\frac{z(t)}{\xi}} \cos [\mathbf{q} \cdot(\mathbf{r}(t)-\mathbf{r}(0))] .
$$

The advantage of using a single walker and following him throughout the computation is that it will sample all the distances $z$ from the wall according to the proper distribution law $c(z)$, as we checked in figure 6 , and the non-uniform concentration profile is automatically accounted for in the simulation.

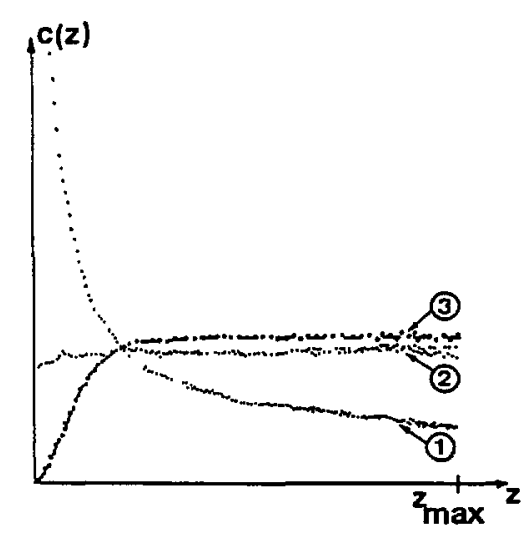

Fig. 6. - Concentration profiles obtained by numerical simulations using in the walker's step only the first term in equation 20 (curve 1), the first two terms (curve 2) and finally all three terms (curve 3). The dashed lines indicate the analytical concentration profile deduced from the Boltzman distribution using equations (9) plus (10) for the particles/wall interaction.

Note that figure 6 illustrates the importance of all three terms in equation (20). Curve 1 shows that accounting for the hydrodynamic repulsion by merely reducing the Brownian step as the particle approaches the wall leads to an accumulation of particles in the vicinity of the wall. It is only by adding the second term (curve 2) that the expected " flat " concentration profile is obtained. The last curve $\left(\mathrm{n}^{\circ} 3\right)$ in the presence of the static interaction potential is well fitted by the Boltzman distribution using equation (9) plus (12) for the particles/wall interaction.

Finally let us mention that to avoid loosing too much time with the walker sampling a region too far away from the wall (where the illumination intensity is too low to give a significant contribution to the correlation function) we put a fictitious purely reflecting wall at $z=z_{\max }$. This distance was chosen as a good compromise between saving time and avoiding distorsions on the correlation function.

This "simulated" correlation function was then fitted with the theoretical expression (8) found for $g_{s}$ and the best fit yielded the simulated value $D(\xi)$. Repeating this procedure for several values of $\xi$ and salt concentrations allowed us to plot the dashed lines shown in figure 4 and to fill the last column of table $I$.

\section{Results and discussion.}

All the experimental results are summarized in table $I$ for different penetration depths and salt concentrations. 
Table I. - Summary of the experimental and simulation results for $D_{\mathrm{s}} / D_{\mathrm{bulk}}$ at various $[\mathrm{NaCl}]$ concentrations and penetration depths $\xi$.

\begin{tabular}{|c|c|c|c|c|c|}
\hline$[\mathrm{NaCl}](\mathrm{mol} / \mathrm{l})$ & $\begin{array}{c}\text { Penetration } \\
\text { Depth }\end{array}$ & {$\left[\begin{array}{l}D_{\mathrm{s}}\left(10^{-8} \mathrm{~cm}^{2}, \mathrm{~s}\right) \\
\text { (cxperiment) }\end{array}\right.$} & $\begin{array}{c}D_{\mathrm{b}}\left(10^{-8} \mathrm{~cm}^{2} / \mathrm{s}\right) \\
\text { (experiment) }\end{array}$ & $\begin{array}{c}D_{\mathrm{s}} / D_{\mathrm{b}} \\
\text { (experiment) }\end{array}$ & $\begin{array}{c}D_{\mathrm{s}} / D_{\mathrm{b}} \\
\text { (simulation) }\end{array}$ \\
\hline No salt added & $\begin{array}{l}\xi=0.85 \mu \mathrm{m} \\
\xi=0.43 \mu \mathrm{m} \\
\xi=0.20 \mu \mathrm{m}\end{array}$ & $\begin{array}{l}4.24 \pm 0.12 \\
4.35 \pm 0.11 \\
4.35 \pm 0.11\end{array}$ & $4.49 \pm 0.04$ & $\begin{array}{l}0.95 \\
0.93 \\
0.97\end{array}$ & $\begin{array}{l}1.0 \\
0.95\end{array}$ \\
\hline $2.5 \times 10^{-3}$ & $\begin{array}{l}\xi=0.85 \mu \mathrm{m} \\
\xi=0.20 \mu \mathrm{m}\end{array}$ & $\begin{array}{l}4.26 \pm 0.12 \\
3.94 \pm 0.04\end{array}$ & $4.62 \pm 0.02$ & $\begin{array}{l}0.92 \\
0.85\end{array}$ & $\begin{array}{l}0.85 \\
0.75\end{array}$ \\
\hline $5.0 \times 10^{-3}$ & $\begin{array}{l}\xi=0.85 \mu \mathrm{m} \\
\xi=0.20 \mu \mathrm{m}\end{array}$ & $\begin{array}{l}4.10 \pm 0.12 \\
3.85 \pm 0.04\end{array}$ & $4.77 \pm 0.10$ & $\begin{array}{l}0.86 \\
0.81\end{array}$ & $\begin{array}{l}0.85 \\
0.74\end{array}$ \\
\hline $7.5 \times 10^{-3}$ & $\begin{array}{l}\xi=0.85 \mu \mathrm{m} \\
\xi=0.20 \mu \mathrm{m}\end{array}$ & $\begin{array}{l}4.05 \pm 0.02 \\
3.78 \pm 0.04\end{array}$ & $4.76 \pm 0.06$ & $\begin{array}{l}0.85 \\
0.79\end{array}$ & $\begin{array}{l}0.85 \\
0.73\end{array}$ \\
\hline $1.0 \times 10^{-2}$ & $\begin{array}{l}\xi=0.85 \mu \mathrm{m} \\
\xi=0.43 \mu \mathrm{m} \\
\xi=0.20 \mu \mathrm{m}\end{array}$ & $\begin{array}{l}4.09 \pm 0.01 \\
3.76 \pm 0.08 \\
3.77 \pm 0.04\end{array}$ & $4.80 \pm 0.06$ & $\begin{array}{l}0.85 \\
0.79 \\
0.79\end{array}$ & $\begin{array}{l}0.85 \\
0.78 \\
0.73\end{array}$ \\
\hline
\end{tabular}

The experiments were conducted as follows : the latex suspensions were prepared in pure water, and the bulk diffusion coefficient was measured. The optical set-up was then aligned for a given penetration depth $\xi$, and the corresponding surface diffusion coefficient measured. Without changing the optics, the necessary salt solution was added and $D_{\mathrm{s}}$ measured at different salt concentration. Changing back the incident angle to $\theta_{i}<\theta_{c}$, the bulk diffusion coefficient was measured on suspensions containing the same salt concentration. Note that the bulk diffusion coefficient $D_{\mathrm{b}}$ is larger in the salty solution than in pure water. This is simply due to the fact that the dangling chains covering the external latex surface carry $\mathrm{OH}^{-}$groups at their tips. In pure water, these chains are more or less fully extended, so as to spread the $\mathrm{OH}^{-}$groups as far apart as possible, whereas in the presence of salt, they can fold back to a more compact structure which allows the particles to have a larger diffusion coefficient [14].

The ratios $D_{\mathrm{s}}(\xi) / D_{\text {bulk }}$ obtained from the experiments are summarized the next to last column of table I and the data extracted from the simulations are indicated in the last column of that table. As expected, we note that the experimentally measured ratio $D_{\mathrm{s}}(\xi) / D_{\text {bulk }}$ increases as the salt concentration is decreased, but that the values obtained in pure water are still significantly lower than 1 . The simulations of « pure water " given at the beginning of the last column were made assuming $[\mathrm{NaCl}]=10^{-6} \mathrm{~mol} / \mathrm{l}$.

As experiments and simulations are in fairly good agreement, we feel confident that the Brownian dynamic simulations will be quite valuable to interpret light scattering data from " hindered » scatterers, such as particles trapped in a porous media or a gel ; it is expected that the correlation function of the electric field scattered by such particles will decay with a time constant reflecting both : 
i) the geometrical constraints which reduce the span of the particles' diffusive motion [15] and

ii) the physical interactions between the particles and the walls, which slow down the particles' Brownian motion itself.

To summarize, we have shown that the Evanescent Quasielastic Light Scattering Technique is a sensitive tool for measuring Brownian dynamics in the immediate vicinity of a rigid surface.

A net decrease of the measured diffusion coefficient is observed, due to the hydrodynamic slowing down of the particles very close to the wall. This effect is more observable when the particles are allowed to get closer to the wall, i.e. when the range of the static wall/particle repulsive interaction decreases.

This method could also prove to be very sensitive to the onset of particles aggregation on the surface, introducing in the computation a "residence time " during which a given particle would remain stuck on the wall before drifting again in the suspension. Simulations are currently being done to test this possibility.

\section{Acknowledgments.}

The authors acknowledge stimulating discussions with Bruce Ackerson and Didier Sornette and wish to thank Pierre Bezot for his valuable help in the experimental part, and $C$. Vanneste and J.P. Roustan for their friendly help in the writing of the computer programs.

\section{References}

[1] See for example BatcheloR G. K., J. Fluid. Mech. 74 (1976) 1.

[2] AdAMCZyK Z. and VAN DE VeN T. G. M., J. Colloid Interface Sci. 84 (1981) 497.

[3] EMAK D. L. and MCCammon J. A., J. Chem. Phys. 69 (4) (1978) 1352.

[4] Clark A. T., Lal M. and Watson G. M., Faraday Discuss. Chem. Soc. 83 (1987) 179.

[5] ADAMCZYK M. and VAN DE VeN T. G. M., J. Colloid. Interface Sci. 96 (1983).

[6] Prieve D. C. and Frej N. A., Langmuir 6 (1990) 396-403.

[7] Thomson N. L., Burghart T. P. and AXelrod D., Biophys. J. 33 (1981) 435.

[8] Aussere D., Hervet H. and Rondelez F., Phys. Rev. Lett. 54 (1985) 1948.

[9] Lan K. H., Ostrowsky N. and Sornette D., Phys. Rev. Lett. 57 (1986) 17.

[10] Bell G. M., Levine S. and McCarmney L. N., J. Coll. Inter. Sci. 33 (1970) 3, 335-359.

[11] Hamaker H. C., Physica 4 (1937) 1058.

[12] FAXeN H., Arkiv. Mat. Astron. Fys. 17 (1923) 27.

[13] Brenner H., Chem. Eng. Sci. 16 (1961) 242.

[14] Trotter C. M. and Pinder D. N., J. Chem. Phys. 75 (1981) 1.

[15] Celestini F., LOBRy L. and Ostrowsky N., to be published. 